\title{
Simulation-based uncertainty correlation modeling in reliability analysis
}

Proc IMechE Part O:

J Risk and Reliability

2018, Vol. 232(6) 725-737

(c) IMechE 2018

Article reuse guidelines:

sagepub.com/journals-permissions DOI: 10.1 | 777/I748006 X18758720

journals.sagepub.com/home/pio

(S)AGE

\author{
Faramarz Khosravi' $\mathbb{D}$, Malte Müller', Michael Glaß ${ }^{2}$ and Jürgen Teich'
}

\begin{abstract}
Due to destructive effects like temperature and radiation, today's embedded systems have to deal with unreliable components. The intensity of these effects depends on uncertain aspects like environmental or usage conditions such that highly safety-critical systems are pessimistically designed for worst-case mission profiles. These uncertain aspects may affect several components simultaneously, implying correlation across uncertainties in their reliability. This paper enables a state-of-the-art uncertainty-aware reliability analysis technique to consider multiple arbitrary correlations; in other words, components' reliability is affected by several uncertain aspects to different degrees. This analysis technique combines reliability models such as binary decision diagrams with a Monte Carlo simulation, and derives the uncertainty distribution of the system's reliability with insights on the mean, quantile intervals, and so on. The proposed correlation method aims at generating correlated samples from the uncertainty distribution of components' reliability such that the shape and statistical properties of each individual distribution remain unchanged. Experimental results confirm that the proposed correlation model enables the employed uncertainty-aware analysis to accurately calculate uncertainty at system level.
\end{abstract}

\author{
Keywords \\ Reliability analysis, uncertainty correlation modeling, Monte Carlo simulation, system-level design
}

Date received: 10 November 2016; accepted: 15 January 2018

\section{Introduction}

The continuous scaling of technology allows for the production of compact high-performance electronic components at low cost. However, their small device structures are increasingly susceptible to destructive effects like radiation or temperature, resulting in inherently unreliable components. This renders reliability one of today's main challenges in the design of embedded systems which require the composing of reliable systems from such unreliable components. ${ }^{1}$

Existing reliability analysis techniques typically assume that the components' reliability is accurately specified, that is, specified as a single value representing its failure probability or rate, and thus, derive a single value for the system's reliability. Nonetheless, manufacturing tolerances as well as environmental and usage conditions have a growing and significant impact on the components' failure rates which may not be known a priori; in other words, they are uncertain and must be specified using probability distributions. As an example, higher temperatures lead to vastly different rates of negative bias temperature instability (NBTI) and oxide breakdown degradations in MOSFET devices. ${ }^{2}$ The variations in components' reliability propagate to the system, and necessitate specifying the system's reliability with an uncertainty distribution as well. In the design of highly safety-critical systems, worst-case mission profiles describe these conditions pessimistically such that the systems are over-designed. To exemplify, an implementation of an H.264 encoder/decoder where hardware components, that is, processing cores and accelerators, are exposed to varying conditions, modeled as variations of their failure rates, is investigated.

\footnotetext{
'Hardware/Software Co-Design, Friedrich-Alexander-Universität Erlangen-Nürnberg (FAU), Erlangen, Germany

${ }^{2}$ Institute of Embedded Systems/Real-Time Systems, Ulm University, Ulm, Germany
}

\section{Corresponding author:}

Faramarz Khosravi, Friedrich-Alexander-Universität Erlangen-Nürnberg (FAU), Chair of Computer Science 12 (Hardware/Software Co-Design), Cauerstraße II, Erlangen 91058, Germany.

Email: faramarz.khosravi@fau.de 
Figure 1 shows the resulting distribution of the system's mean time to failure (MTTF). (Note that MTTF is a scalar value equal to the expected value of the system's time to failure distribution, if components' failure rates are given as scalar values. However, given an uncertainty distribution for components' failure rates, different values can be calculated for the system's MTTF which constitute a probability distribution as shown in Figure 1. See section "Uncertainty-aware reliability analysis" for more details.) It includes the best and worst cases as well as the $95 \%$ quantile interval, that is, the interval between the $2.5 \%$ and $97.5 \%$ quantiles, of the system's MTTF derived with the uncertainty-aware analysis technique presented by Khosravi et al. ${ }^{3}$ For the dashed curve, there exists a significant difference between the bounds and the relevant scenarios, namely the quantile interval. Considering quantiles with a high confidence, rather than the best and worst cases, shows great potential for designing systems less pessimistically.

Destructive effects like temperature may affect several adjacent components at a time ${ }^{4,5}$ and render their uncertainties correlated. Consider again Figure 1 and the solid curve: the components are exposed to the same ambient temperature and the resulting distribution and quantiles are significantly different from those where no correlation in the uncertainty of the components' reliability was assumed (dashed).

To analyze the system reliability in the presence of uncertainty we proposed in Khosravi et al. ${ }^{3}$ to model system components using reliability functions with parameters given as probability distributions. Such distributions may be approximated by for example Gaussian variations around a mean derived from measurements or field data, or obtained by techniques at lower abstraction levels. ${ }^{6,7}$ In the latter case, uncertain parameters can follow correlated, non-Gaussian distributions. ${ }^{8}$ Therefore, the uncertainty model in Khosravi et al. ${ }^{3}$ is not restricted to any specific distribution type, but can handle different distributions including sample data. Furthermore, to deal with correlated uncertainty, it classifies the components which are exposed to the same correlation source, that is, coupling mechanism, into a correlation group. In order to be capable of considering uncertain characteristics and their different correlations, it combines a formal analysis core with a Monte Carlo (MC) simulation. (MC simulation is the most straightforward approach to analyzing stochastic systems, ${ }^{9}$ and is used in this work due to its generality and ease for consideration of correlation among probabilistic variables in the analysis.) While the analysis core can be realized by almost any existing technique, such as binary decision diagrams (BDDs), fault trees, Markov chains, and so on, the simulation enables an extensible and affordable consideration of uncertainties with controllable execution time overhead.

Nevertheless, the correlation model proposed in Khosravi et al. ${ }^{3}$ suffers from limitations resulting in a lack of flexibility and generality due to the assumptions that (i) each component is either independent or is a

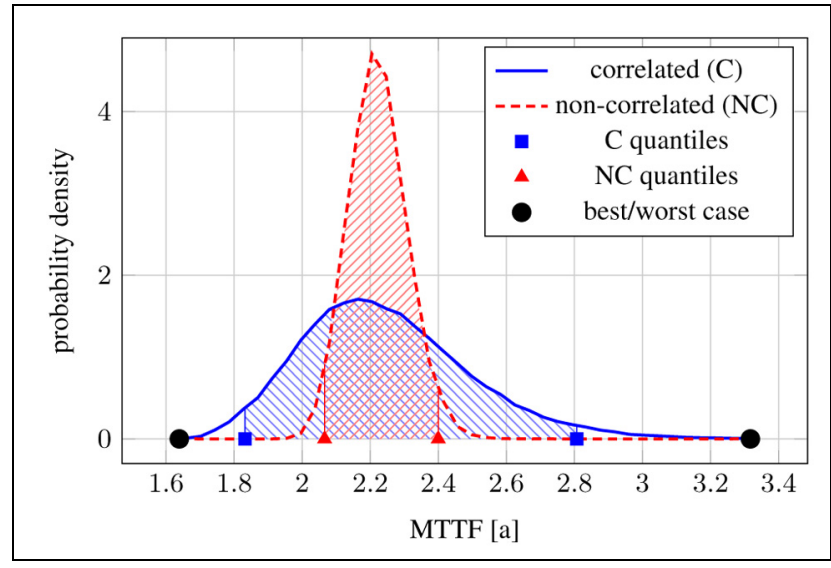

Figure I. The distribution of the MTTF of an implementation candidate of an H.264 encoder/decoder: the solid curve shows the distribution for components with a correlated uncertainty, whereas the distribution for independent components is given by the dashed curve. To highlight the significance of uncertainty and correlation consideration, theoretical bounds as well as $95 \%$ quantiles are depicted. Adapted from Khosravi et al. ${ }^{3}$

member of only one correlation group, that is, its uncertainty is restricted to being affected by at most one source of correlation, and (ii) the components within a correlation group are affected by the corresponding source of correlation to the same extent. In reality, however, the uncertainty of a component can be affected by multiple correlation sources, for example temperature, usage profile, and so on. In addition, a certain source of correlation would affect different components to different extents. For example, the closer a component is to a hot spot, the more impact the temperature has on its reliability. To overcome the above-mentioned limitations, this paper proposes a new correlation model which allows components to be affected to different degrees by multiple correlation sources.

Incorporating more details of uncertainty correlation can significantly enhance our realization of the uncertainty distribution and the statistical properties of the system's reliability. Experiments show that the analysis can obtain statistical properties for the reliability of complex embedded systems at a reasonable overhead.

This work is done based on the following assumptions.

1. The proposed uncertainty-aware analysis is a framework to deal with uncertainty in system-level design. It is sufficiently general to incorporate various analysis techniques, to consider uncertainty in different parameters such as components' failure probabilities, soft error rates, and so on, and to be applicable to different applications and case studies.

2. The uncertainty distributions should be specified by the user or an uncertainty quantification technique. Therefore, our framework allows uncertainty distributions to be from instances of 
standard discrete and continuous probability distributions such as uniform ones, Gaussian ones, beta ones, and so on, up to arbitrary distribution given as a histogram of sample observations.

3. The set of correlation sources as well as whether and to what extent each component is correlated to each correlation source is given.

The rest of this paper is organized as follows: "Related work" reviews the state of the art. "Uncertainty-aware reliability analysis" explains the uncertainty model as well as the proposed uncertaintyaware reliability analysis while "Modeling of uncertainty correlation" introduces the uncertainty correlation and presents the proposed advanced correlation model. "Experimental setup and evaluations" presents the experimental setup and evaluation results, and finally, "Conclusion" concludes this work.

\section{Related work}

In the context of probabilistic risk assessment, uncertainty is classified into two main categories, namely, aleatory and epistemic uncertainties. The former is defined as "inherent variation associated with the physical system or the environment under consideration" 10 and the latter describes the uncertainty resulting from "any lack of knowledge or information in any phase or activity of the modeling process". ${ }^{10}$ In reliability analysis, aleatory uncertainty may refer to the stochastic nature of environmental and operational conditions ${ }^{11-}$ 13 as well as failure and degradation mechanisms, 6,7 whereas the epistemic uncertainty refers to the estimations made due to modeling complexities and testing restrictions. The epistemic uncertainty can be due to (i) difficulties accurately quantifying, monitoring, or sensing characteristics like chip temperature, ${ }^{11-13}$ time to failure, ${ }^{2}$ error rate, ${ }^{14}$ and so on, at runtime; (ii) temporal or experimental restrictions in component testing; ${ }^{15}$ and (iii) inaccuracy in modeling the transformation or propagation of errors across different design abstraction levels. ${ }^{16}$ Indeed, the uncertainty to be dealt with in system-level design has both aleatory and epistemic elements which cannot be easily differentiated. Therefore, in this work, we assume the effects of various uncertainty sources are aggregated and represented by probability distributions.

The existing work in the context of uncertaintyaware design can be classified into three groups that, respectively, aim at (i) extracting uncertainty distributions of components as well as the correlation among them through observing variations in design parameters, ${ }^{2,4-6,12,14,17}$ (ii) deriving uncertainty at system level assuming that the uncertainty in analysis models or input parameters is estimated a priori, ${ }^{18-22}$ and (iii) optimizing the system with respect to the estimated uncertainty in its design objectives. ${ }^{23,24}$

To quantify uncertainty, Kaczer et al. ${ }^{6}$ investigate the effects of technology scaling on the variability of
NBTI-based degradations in the threshold voltage. Considering a lifetime criterion as an upper bound for these degradations, they realize the deviations from the device's expected lifetime. Karl et al. ${ }^{2}$ show that variations in oxide dimensions, temperature and supply voltage can change the probability of oxide breakdown mechanism, and therefore significantly affect the device's time-to-failure distribution. Ankireddi ${ }^{12}$ presents a method to predict hot-spot temperature based on the heat gradient between cores and on-chip sensors, and reports uncertainty through histograms of thermal gradients considering maximum sensing errors. Zhang et al. ${ }^{13}$ assume that components' power densities are uncertain due to workloads, device parameters and environmental effects such as ambient temperature, and derive the correlation among these uncertainties. They combine this design-time information with runtime temperature readings from on-chip sensors to estimate the expected value of the operational temperature through the chip. Janicki et al. ${ }^{4}$ show that technology scaling results in a more even distribution of temperature throughout the whole chip with an up to $65 \%$ steady-state thermal coupling among cores, and observe that more than $80 \%$ of the temperature rise in a hot spot is due to external factors rather than the core's self-heating. Thermal correlation can be even more significant among components in a vertical neighborhood in three-dimensional integrated circuits. ${ }^{5}$ Souza et al. ${ }^{17}$ estimate the failure rate of a system exposed to various environmental disturbances, for example high ambient temperature, and capture the variations among the failure rates estimated according to different reliability standards such as IEC $61709 .{ }^{25}$ Chen et al. ${ }^{14}$ propose an adaptive confidence-driven computing approach to provide a certain reliability at the system level by runtime monitoring of error rate fluctuations and by applying fine-grained temporal and spatial redundancies at circuit level. In this paper, we assume parameter uncertainty is estimated using such methods and may follow any distribution with arbitrary correlations.

Traditionally, uncertainty-aware analysis is performed mathematically to predict the distribution of uncertain objectives from variations in input parameters. Yin et al. ${ }^{18}$ consider the uncertainty in the failure rates of the system's components, and derive mean and quantiles of the system's reliability using secondorder and Gaussian approximations. However, the application of these mathematical methods, compared to $\mathrm{MC}$ simulation, is limited to a few reliability models, certain distribution types and non-correlated uncertain parameters. Probability bounds ${ }^{26}$ is a common approach to deriving the uncertainty interval in the output of a model or function, given the uncertainty interval in its components or input parameters. It decomposes the uncertainty of each of the $n$ uncertain parameters into $m$ subintervals with equal probability, called focal elements. It then evaluates the model with all $m^{n}$ possible combinations of focal elements. This approach is useful when limited information on the 
input uncertainty distributions is available. ${ }^{26}$ Otherwise, MC simulation provides a more straightforward implementation and delivers less pessimistic estimates of the output uncertainty. Taking a similar approach, Sallak et al. ${ }^{21}$ propose a belief model for considering uncertainty during the mathematical reliability analysis of series-parallel systems. They model uncertainty as log-normal variations in the components' failure probabilities, and also consider a probability for having no clue whether a component is operating or has failed. Nonetheless, their belief model is only applicable to log-normal uncertainties, and leads to a significant error of up to $33 \%$ in the uncertainty bounds of the system's reliability compared to a relatively accurate MC-based approach. ${ }^{21}$ The work by Pedroni and $\mathrm{Zio}^{27}$ extends the belief model to consider perfect and unknown correlation among uncertainty intervals of the system's components. Assuming that sufficient information on the uncertainty distributions and their correlation is available, we instead use MC simulation which enables more accurate and less conservative correlation modeling in uncertainty analysis.

The work by Esfahani et al. ${ }^{19}$ deals with uncertainty as the lack of knowledge of the exact effects of architectural decisions on objective values, and models it using a triangular fuzzy representation including its anticipated, optimistic and pessimistic values. Meedeniya et al. ${ }^{20}$ propose a reliability evaluation method to deal with non-correlated uncertainty distributions in components' reliability. Their uncertainty analysis is based on sampling from uncertain parameters, and evaluating the system with sampled parameters to obtain statistical information, for example percentiles. Unlike their work, ${ }^{20}$ our previous work ${ }^{3}$ takes into account the correlations between the components' failure rates while sampling.

To deal with uncertain objectives during a multiobjective optimization, Teich $^{23}$ compares implementation candidates with uniformly distributed objectives by calculating the probability that one implementation dominates the other. Limbourg ${ }^{24}$ extends the concepts of strong and weak dominance by considering lower and upper bounds instead of point estimates. To limit the inaccuracy and delay of comparison, he incorporates the weak dominance operator in the parent selection and the strong dominance operator in the solution archive. However, these methods cannot handle arbitrariness in the uncertainty distributions. In Khosravi et al. ${ }^{3}$ we proposed a comparison operator that compares various statistical properties of distributions and enables us to consider arbitrarily distributed design objectives.

The work at hand focuses on analyzing uncertainty in the system's reliability assuming that the uncertainty distributions of the components' reliability and the correlation among them are available. It generalizes the uncertainty analysis in Khosravi et al. ${ }^{3}$ considerably by providing a more realistic modeling of the correlations between the uncertain characteristics of different components.

\section{Uncertainty-aware reliability analysis}

In this section, we introduce the proposed uncertaintyaware reliability analysis approach. First, we give an outline of the overall flow, and then we present the modeling of components' uncertainties.

Overall analysis flow. The general idea of the proposed analysis is given in Figure 2. At its core, the approach may employ various existing reliability analysis techniques that typically require a reliability function $\mathcal{R}_{r}(t)$ of each component $r$ at time $t$, and deliver the system implementation's reliability function $\mathcal{R}_{\text {imp }}(t)$. The standard definition of $\mathcal{R}(t)$ with $\mathcal{R}: \mathbb{R}_{0}^{+} \rightarrow[0,1], \mathcal{R}(0)=1$, $\lim _{t \rightarrow \infty} \mathcal{R}(t)=0$, and $\forall t, t^{\prime} \in \mathbb{R}_{0}^{+}, t \leqslant t^{\prime}: \mathcal{R}(t) \geqslant \mathcal{R}\left(t^{\prime}\right)$ is assumed. In the concrete case, a BDD-based approach is shown, modeling an implementation consisting of two components in series. This formal analysis core is employed by a MC-based sampler which samples reliability functions from each component and applies them to the analysis core. The gathered sample reliability function $\mathcal{R}_{\text {imp }}^{s}(t)$ of the $s$ th sample is collected by a statistical simulator that constructs the reliability uncertainty distribution of the system implementation as a set of sampled reliability functions $\Phi_{\text {imp }}=$ $\bigcup_{s=1}^{n}\left\{\mathcal{R}_{\text {imp }}^{s}(t)\right\}$. The statistical simulator determines the number of required samples $n$ to later obtain desired statistical properties like mean and quantiles from $\Phi_{\text {imp }}$ with a required confidence level; see. To exemplify, for each sample $\mathcal{R}_{\text {imp }}^{s}(t)$ in $\Phi_{\text {imp }}$, the MTTF can be calculated via the following integration:

$$
\operatorname{MTTF}_{s}=\int_{0}^{\infty} t \cdot f^{s}(t) \mathrm{d} t \text { with } f^{s}(t)=\frac{\mathrm{d}\left(1-\mathcal{R}_{\text {imp }}^{s}(t)\right)}{\mathrm{d} t}
$$

The integration is performed using Romberg's method $^{28}$ which is an iterative technique to calculate the area below the function's curve. This algorithm continues until the increase in the accuracy achieved by the last iteration becomes smaller than a threshold value called the stopping criterion. The smaller the stopping criterion amount, the more iterations this method requires but the more accurately it performs the integration. Based on each sample's MTTF, design objectives such as the best-, worst-, and average-case MTTFs and so on can be derived. Note that the consideration of MTTF as a system-wide reliability metric is merely intended to show the ability of our framework to incorporate complex and time-dependent reliability analyses.

Uncertainty modeling. We propose to model the reliability characteristics in the presence of uncertainty $\mathcal{U}_{r}$ of a component $r$ by reliability functions $\mathcal{R}_{r}(t)$ that are distributed within given lower bound $\mathcal{R}_{r}^{l}(t)$ and upper bound $\mathcal{R}_{r}^{u}(t)$ reliability functions,

$$
\mathcal{U}_{r}=\left[\mathcal{R}_{r}^{l}(t), \mathcal{R}_{r}^{u}(t)\right]
$$

In particular, the outlined sampler takes $\mathcal{U}_{r}$ as input and delivers a sampled reliability function $\mathcal{R}_{r}^{s}(t)$ for 


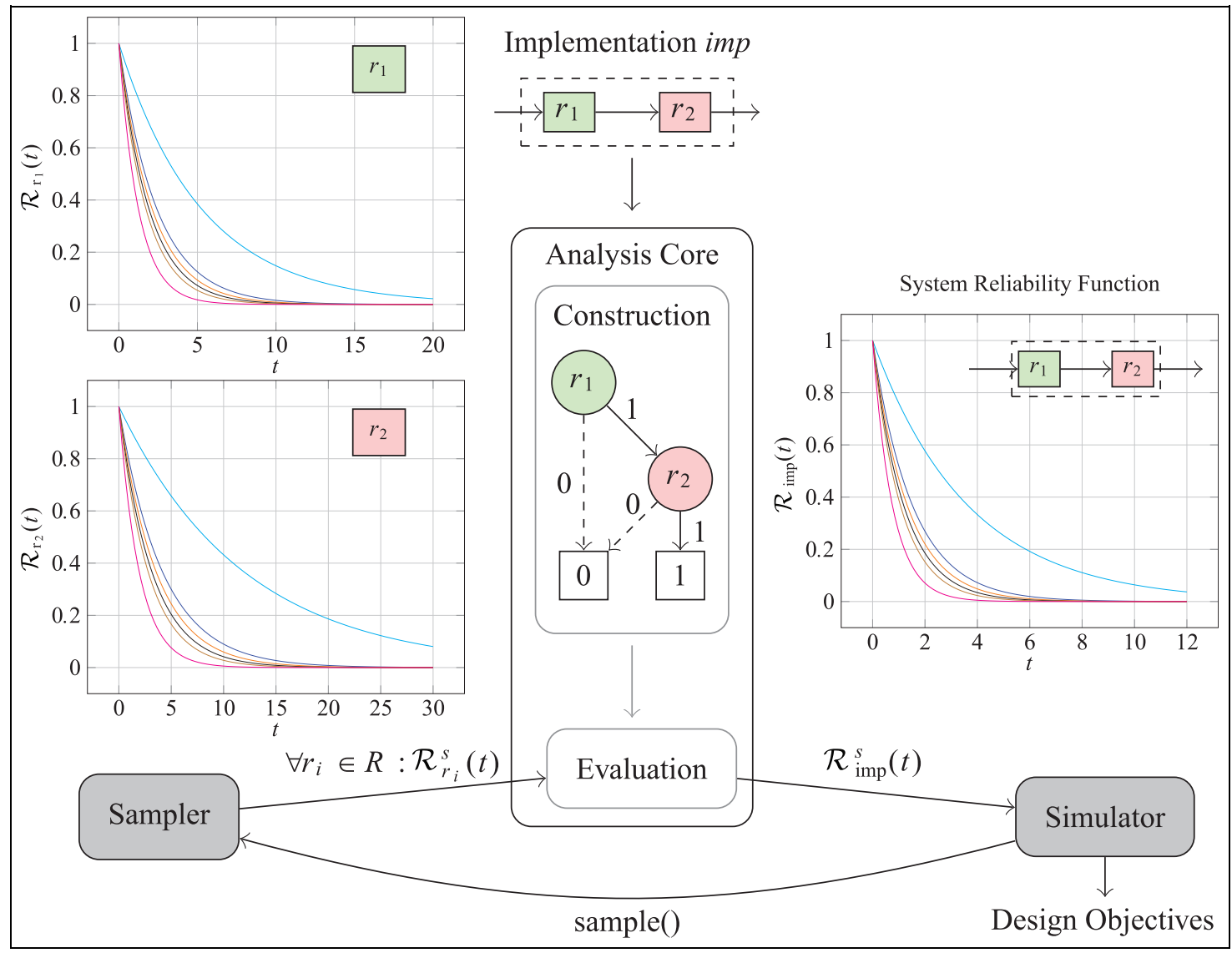

Figure 2. The proposed uncertainty-aware reliability analysis. The sampler applies samples of reliability functions $\mathcal{R}(t)$ for each component $r_{i}$ in the implementation imp to the existing reliability analysis core: here, a BDD. The core constructs a structure function representing imp, and re-evaluates it for the sampled reliability functions of the components, $\mathcal{R}_{r_{i}}^{s}(t) s$, in order to provide samples for the reliability functions of imp, $\mathcal{R}_{\text {imp }}(t)$. The desired statistical properties are then obtained for the design objectives and sent to the optimization. Adapted from Khosravi et al. ${ }^{3}$

which $\forall t \in \mathbb{R}_{0}^{+}: \mathcal{R}_{r}^{l}(t) \leqslant \mathcal{R}_{r}^{s}(t) \leqslant \mathcal{R}_{r}^{u}(t)$ holds. Note that the sampler has to ensure that the sampled reliability functions follow the intended distribution within the given bounds. It enables the consideration of arbitrary distributions, in particular the well-known discrete and continuous distributions also used by Meedeniya et al. ${ }^{20}$ like uniform, Gaussian, or beta distributions, to model the uncertainty induced by ambient temperature.

Note that the employed reliability analysis model (here, BDD), is assumed to be exact, and thus uncertainty is considered in the reliability of components only. In the case of simulation-based analysis techniques such as that by Aliee et al., ${ }^{29}$ where re-evaluating the system's reliability with the same inputs may result in different estimations, the model's uncertainty must also be taken into account.

In practice, component reliability is often derived from measurements that are fitted to closed-form reliability functions with exponential $\left(\mathcal{R}_{r}(t)=e^{-\lambda_{r} \cdot t}\right)$ and Weibull $\left(\mathcal{R}_{r}(t)=e^{-\lambda_{r} \cdot t^{\beta_{r}}}\right)$ functions being frequently used. As can be seen, these distributions are parametrized with a failure rate $\lambda_{r}$. Here, the proposed uncertainty model $\mathcal{U}_{r}$ can be refined to employ a set of uncertain parameters $P_{r}$, distributed within the bounds $\left[P_{r}^{l}, P_{r}^{u}\right]$. Given $\left[P_{r}^{l}, P_{r}^{u}\right]$, the sampler takes a sample from each parameter $p_{r} \in P_{r}$, and, together with the assumed reliability function, constructs a sample reliability function $\mathcal{R}_{r}^{s}(t)$. To exemplify, assuming an exponential function with the component's failure rate $\lambda_{r}$ distributed within bounds $\left[\lambda_{r}^{l}, \lambda_{r}^{u}\right]=[0.008,0.009]$, a sample $\lambda_{r}^{s}=0.0088$ could be derived which results in $\mathcal{R}_{r}^{s}(t)=e^{-0.0088 t}$.

\section{Modeling of uncertainty correlation}

To model correlation among the uncertain parameters of the system's components, we investigate whether they are exposed to common uncertainty sources - they form a correlation group - and are, thus, subject to correlative variations. For example, assume two components are fabricated in the same package and are affected by the same ambient temperature while another component is integrated in a separate package. The reliability characteristics of the first two 


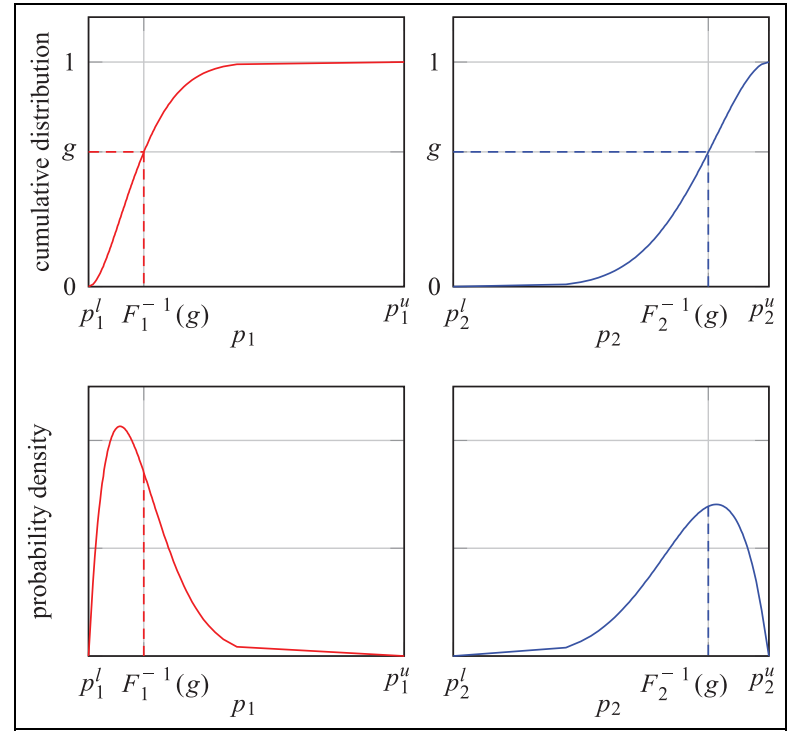

Figure 3. Generating correlated samples for two uncertain parameters $p_{1}$ and $p_{2}$ in a correlation group using the simple correlation model by Khosravi et al. ${ }^{3}$ First, a random quantile probability $g$ is sampled from a uniform distribution between 0 and $I$. Then, the gth quantiles from the probability density functions of $p_{1}$ and $p_{2}$ are returned.

components are considered in a correlation group, but the uncertainty in the reliability of the third component is treated as an independent distribution.

As a concrete example, the work by Zhang et al. ${ }^{13}$ derives the uncertainty distribution and correlations for the power density of different components in a highperformance processor. Given the linear relationship between the power density and the temperature of a component, ${ }^{30}$ the uncertainty distributions and their correlations can be derived for the components' temperatures. The correlation among the temperatures of different components can affect their reliability as many failure and degradation mechanisms are sensitive to device temperature.

Assuming that the uncertainty sources and the correlation groups are given, we first review the simple correlation model ${ }^{3}$ which allows each parameter to be either independent or deterministically subject to only one source of uncertainty, and, thereafter, we overcome the limitations of this model by presenting an advanced correlation model.

Simple correlation model. In this model, as introduced by Khosravi et al., ${ }^{3}$ we aim at generating correlated samples from uncertain parameters using the correlation information, grouping. At each implementation evaluation step, first a random quantile $g$ (uniformly distributed between 0 and 1 ) for each group $G$ is generated that intends to control the sampling process of the components in $G$. While sampling from an uncertain parameter $p$, we check if it is a member of any correlation group or not. If $p$ is a member of group $G$, we return the point from the probability distribution of $p$ at which its cumulative distribution function (CDF) reaches $g$. This point can be obtained using the inverse CDF of $p$ at point $g, F_{p}^{-1}(g)$; see the example shown in Figure 3. Otherwise, a sample is taken independently from the distribution of $p$.

The rationale behind generating a random quantile $g$ for each group $G$ is that (i) we can align and vary the samples of (diversely distributed) uncertain parameters in $G$ according to $g$, (ii) we can keep the distribution of uncertain parameters in $G$ unchanged since $g$ follows a standard uniform distribution, and (iii) we can separate the sampling process of different groups by taking independent samples of $g$ among groups.

The main limitations of the simple correlation model by Khosravi et al. $^{3}$ are that (i) a parameter $p$ can be a member of at most one group $G$, and therefore it cannot share any uncertainty correlations with parameters outside $G$, and (ii) all members of $G$ are correlated to its sample quantiles to the same degree. As an example, a component's temperature might be affected by several heat sources with different intensities. Moreover, the temperatures of different components being exposed to the same heat source may not be equally affected since they are at different distances from it. To overcome these limitations, we propose a novel correlation model in the following section.

Advanced correlation model. In this section, we resolve the limitations of the simple correlation model by Khosravi et al. ${ }^{3}$ by proposing an advanced correlation model. Since we have full control over the sampling process in our MC simulation, we can not only consider uncertain parameters being included in multiple correlation groups, but also specify to what extent they are correlated within each of the groups. Assuming $k$ possible correlation groups are given, the correlation information of an uncertain parameter $p$ is defined as a list of $k$ real values $d_{i} \in[0,+1], 1 \leqslant i \leqslant k$, called the degree of correlation. (Although our correlation model can be adjusted to consider negative correlations, our reliability analysis framework deals with only positive correlations since changes in the intensity of destructive effects, for example heat, make the reliability of different components increase or decrease together.) A nonzero $d_{i}$ implies that $p$ is a member of a correlation group $G_{i}$. Therefore, we define the set $\Delta$ of all groups wherein $p$ is a member as follows:

$$
\Delta=\left\{G_{i} \mid d_{i} \neq 0 \wedge i=1 \ldots k\right\}
$$

To generate a sample $\rho$ from the uncertain parameter $p$, we first generate a sample $\rho_{i}$ for each individual group $G_{i} \in \Delta$ as follows:

$$
\rho_{i}=d_{i} g_{i}+\left(1-d_{i}\right) u
$$

where $u$ is a sample quantile generated independently. It represents the independent effects of the component $r$ on $p$ and is termed uniqueness in this paper. (We assume 
the use of commercial off-the-shelf and intellectual property components that are individually tested, and believe that the proposed correlation model obviates the need for intolerably expensive exhaustive tests of the whole implementation candidate.)

Thus, we calculate the aggregate effects of $\Delta$ on $p$ as follows:

$$
\rho_{T}=\frac{1}{|\Delta|} \sum_{i \mid G_{i} \in \Delta} \rho_{i}
$$

Considering both $u$ and $g_{i}$ as quantile probabilities would lead $\rho_{i}$, in equation (1), and $\rho_{T}$, in equation (2), also to be quantiles which should be transformed using $F_{p}^{-1}$ to outline the distribution of $p$. Based on where we apply this transformation, to $g_{i}$ and $u$, to $\rho_{i}$, or to $\rho_{T}$, the following possibilities exist.

(I) Applying $F_{p}^{-1}$ to $g_{i}$ and $u$ individually:

$$
\rho_{\mathrm{I}}=\frac{1}{|\Delta|} \sum_{i \mid G_{i} \in \Delta}\left(d_{i} F_{p}^{-1}\left(g_{i}\right)+\left(1-d_{i}\right) F_{p}^{-1}(u)\right)
$$

(II) Applying $F_{p}^{-1}$ to $\rho_{i}$ :

$$
\begin{aligned}
\rho_{\mathrm{II}} & =\frac{1}{|\Delta|} \sum_{i \mid G_{i} \in \Delta} F_{p}^{-1}\left(\rho_{i}\right) \\
& =\frac{1}{|\Delta|} \sum_{i \mid G_{i} \in \Delta} F_{p}^{-1}\left(d_{i} g_{i}+\left(1-d_{i}\right) u\right)
\end{aligned}
$$

(III) Applying $F_{p}^{-1}$ to $\rho_{T}$ :

$$
\begin{aligned}
\rho_{\mathrm{III}} & =F_{p}^{-1}\left(\rho_{T}\right) \\
& =F_{p}^{-1}\left(\frac{1}{|\Delta|} \sum_{i \mid G_{i} \in \Delta}\left(d_{i} g_{i}+\left(1-d_{i}\right) u\right)\right)
\end{aligned}
$$

Depending on the original distribution of $p$, these three transformations might result in either similar or significantly different correlated distributions. For example, two independent but similar experiments on two random variables with different distributions are shown in Figures 4 and 5. These experiments depict the effects of the above-mentioned transformations on the original distributions. Note that both experiments use equal correlation groups and degrees, and in each experiment, only the transformation strategy alters. While the correlated distributions shown in Figure 4 are rather similar, those in Figure 5 indicate how different these transformations can act. Although all these transformations keep the mean value of the original, that is, non-correlated, distribution almost unchanged, they result in correlated distributions where more samples are accumulated around the mean. Such accumulation is due to the convolution between $u$ and each effect $g_{i}$ shared within a correlation group, and alters the characteristics of the original distribution. The intensity of this

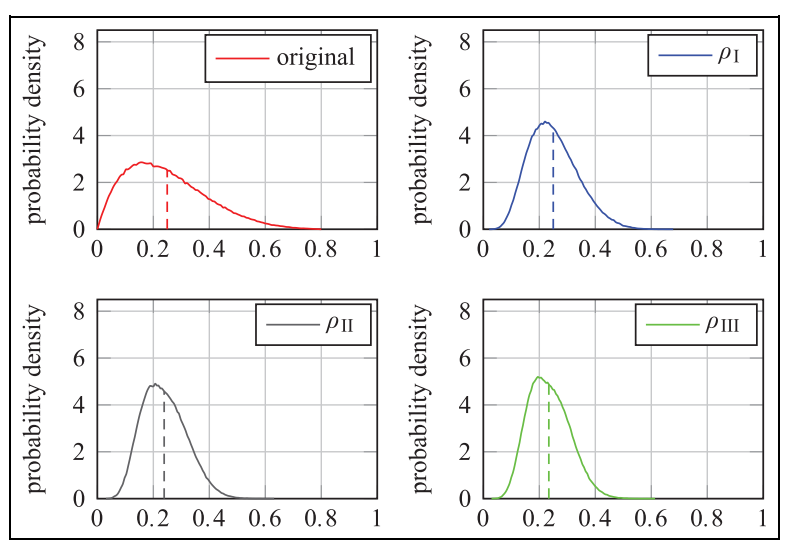

Figure 4. The effects of transformations in equations (3) to (5) on an uncertain parameter $p$ following a beta distribution with parameters $\alpha=2$ and $\beta=6$. The uncertain parameter is considered in three correlation groups with the degrees of $d_{1}=0.2, d_{2}=0.5$, and $d_{3}=0.75$. Despite keeping the mean of the original distribution unchanged, they accumulate the correlated samples around the mean, and thus change the distribution's shape and bounds. Moreover, the first strategy in equation (3) results in a more symmetric shape because it converts quantiles, $g_{i}$ and $u$, to samples from the original distribution before applying the weighted sum and averaging operations.

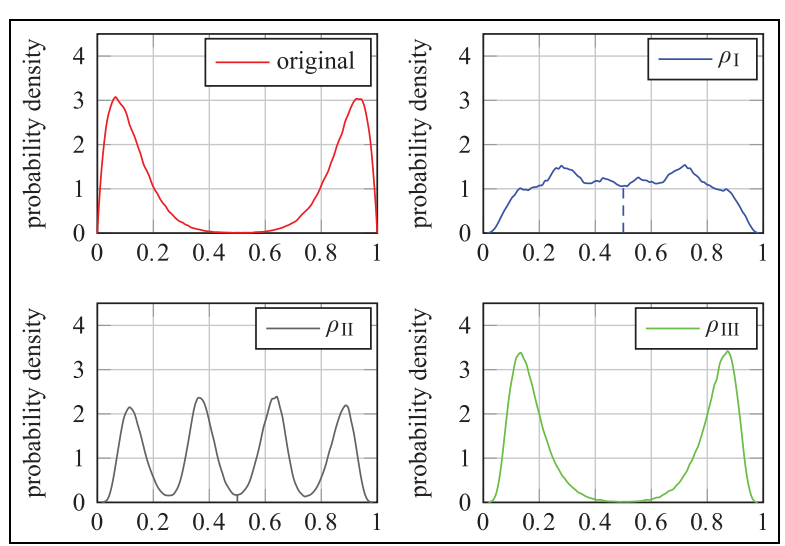

Figure 5. The effects of transformations in equations (3) to (5), with the same grouping information as in Figure 4, on an uncertain parameter. The shape of this original distribution leads to very different distributions for the correlated samples taken using the three strategies.

phenomenon depends on the original distribution, the number of groups, and the degrees of correlation. For higher correlation degrees, the weighted sum in equation (1) would lead to less uniqueness. Moreover, increasing the number of groups increases the effects of averaging in equation (2), and results in a normal distribution (see Appendix 2 for further considerations).

As shown in Figure 5, there exist contrasts between the correlated distributions derived by the aforementioned strategies due to different applications of $F_{p}^{-1}$ in equations (3) to (5). Equation (3) first transforms $g_{i}$ and $u$ to samples of the original distribution using $F_{p}^{-1}$, and then applies a weighted sum, equation (1), and averaging, equation (2). Since $F_{p}^{-1}\left(g_{i}\right)$ and $F_{p}^{-1}(u)$ can be 


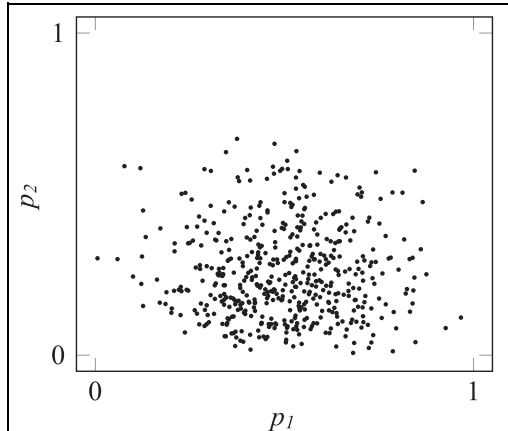

(a) No correlation

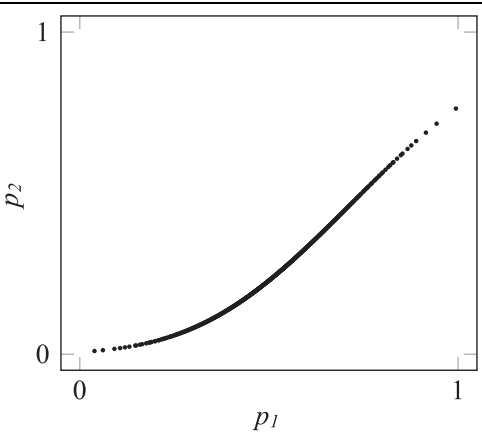

(b) Simple correlation

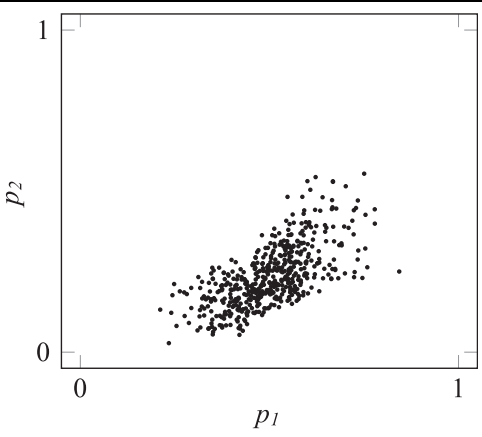

(c) Advanced correlation

Figure 6. Scatter plot showing the distribution of two correlated uncertain parameters $p_{1}$ and $p_{2}$ which are in one correlation group to the degrees of 0.7 and 0.5 , respectively; $p_{1}$ follows a Gaussian distribution with $\mu=0.5$ and $\sigma=0.16$, and $p_{2}$ is specified by a beta distribution with $\alpha=2$ and $\beta=6$. Whereas applying no correlation results in independent samples and the simple model generates completely correlated samples, the advanced correlation model can realistically consider the existing correlation between $p_{1}$ and $p_{2}$ while sampling. Note that in the advanced model, $p_{1}$ and $p_{2}$ are not explicitly correlated to each other, but the correlation between them is implied by their individual correlations to the shared uncertainty source.

from either ridge in the original distribution, they will often compensate each other through these operations, resulting in samples of $\rho_{\mathrm{I}}$ accumulating around the mean of $u$. Nonetheless, equation (4) applies the weighted sum operation to quantiles, $g_{i}$ and $u$, before deriving samples from the original distribution using $F_{p}^{-1}$. Although it results in the convolution of quantile values, the resulting distribution keeps the characteristics of the original distribution. (The convolution of quantile probabilities as random variables uniformly distributed between 0 and 1 is discussed in Appendix 2.) However, applying the averaging in equation (2) accumulates samples to form new ridges whose number and positions depend on the grouping information. Unlike the first two strategies, the third transformation in equation (5) applies both operations in the quantile space, and thus converts the correlated quantile to the corresponding value of the distribution using $F_{p}^{-1}$. It is also worth noticing that the weighted sum and averaging operations always result in correlated samples within the bounds of the original distribution of the uncertain parameter $p$. Given this observation, we adopt $\rho_{\text {III }}$ in the rest of our experiments since it keeps the probability density function ( PDF) of uncertain parameters.

To illustrate the superiority of the advanced correlation model in generating correlated samples, Figure 6 plots the distributions of two uncertain parameters sampled considering no correlation and according to the simple and advanced correlation models. It shows that the advanced correlation model successfully incorporates the existing correlation between uncertain parameters into the sampling.

\section{Experimental setup and evaluations}

In this section, we discuss the experiments for evaluating the accuracy and the execution time overhead of our proposed uncertainty-aware reliability analysis. Table 1 lists three high-level synthesis test problems
Table I. The test problems used in our experiments.

\begin{tabular}{lllll}
\hline Problem & Resources & Tasks & Mappings & $k$ \\
\hline H.264 & 15 & 66 & 275 & 3 \\
Synth-I & 25 & 56 & 261 & 5 \\
Synth-II & 50 & 101 & 592 & 5 \\
\hline
\end{tabular}

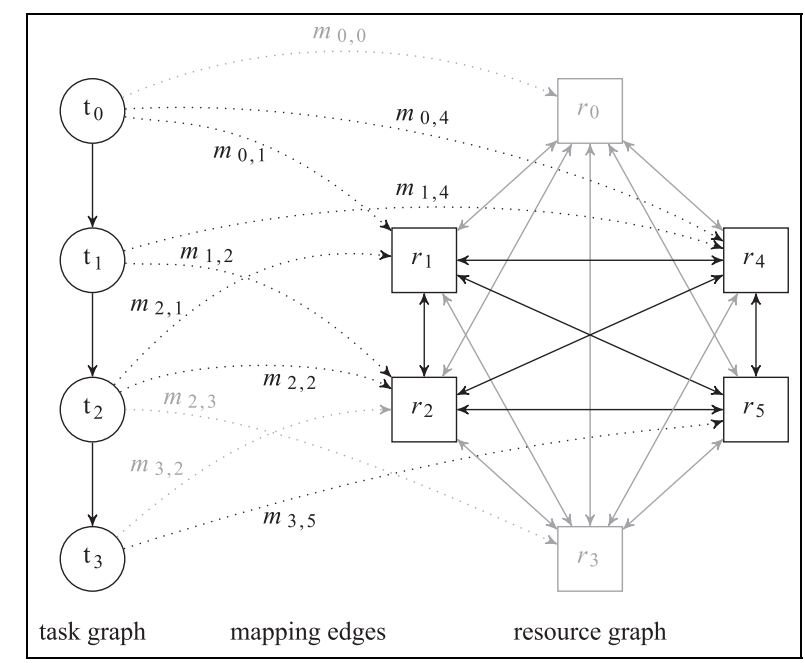

Figure 7. A problem specification including a task graph, a resource graph and a set of possible task-to-resource mappings. In the task graph, the edge from $t_{i}$ to $t_{j}$ indicates a data dependency from $t_{i}$ to $t_{j}$, while in the resource graph, the edge from $r_{i}$ to $r_{j}$ shows a dedicated communication line from $r_{i}$ to $r_{j}$. A mapping edge $m_{i, j}$ represents a mapping from the task $t_{i}$ to the resource $r_{j}$. Shown in black is an implementation candidate obtained by resource allocation and task binding.

including a real-world specification for an H.264 encoder/decoder as well as two synthetic specifications of different sizes and complexities. It summarizes each problem's characteristics including the number of resources, tasks, task-to-resource mappings, and correlation groups, that is, the number of groups wherein 
resources are subject to the same uncertainty sources. As an example, Figure 7 shows the specification of a test problem including four communicating tasks $\mathrm{t}_{i}$, $i \in[0, \ldots, 3]$, six resources $r_{j}, j \in[0, \ldots, 5]$, and ten mappings $m_{i, j}$ from $t_{i}$ to $r_{j}$.

Implementation candidates can be derived from the system specification through system-level synthesis ${ }^{31}$ which consists of resource allocation, task binding, and scheduling steps. Resource allocation selects a subset of resources that can host a subset of possible task instances including at least one instance of each task. Task binding binds at least one instance of each task to the allocated resources through activating the corresponding task-to-resource mappings. Scheduling seeks a feasible start time for each task instance and aims at minimizing the overall execution time of the task graph. An implementation is feasible if certain timing and resource utilization constraints are fulfilled. The parts of the example specification in Figure 7 that are shown in black specify the resource allocation and task binding of an example implementation candidate. More details on the underlying system synthesis and design space exploration (DSE) can be found in the work by Blickle et al. ${ }^{31}$ and Streichert et al. ${ }^{32}$

The analysis core used in our experiments is a stateof-the-art BDD-based reliability analysis technique ${ }^{33}$ that can handle complex system structures considering features like resource sharing, and so on. All experiments are carried out on a desktop computer with a $3.40 \mathrm{GHz}$ CPU.

Several experiments are performed to evaluate the proposed framework in the following subsections. First, we address the accuracy and confidence of the uncertainty-aware reliability analysis and report its execution time overheads. Then, we discuss the effects of ignoring or simplifying uncertainty correlation modeling on the quality of reliability analysis.

Confidence adaptation. The statistical properties of the uncertain objectives derived through MC simulation should guarantee a certain confidence level to enable robust design decisions and objective comparisons during the DSE. Given a required confidence level of $(1-\alpha) \cdot 100 \%$ and a minimum number of samples $n_{\min }$, the maximum error $e_{\max }$ can be determined which gives a confidence interval for the precise mean value $\mu, \mu \in\left[\hat{\mu}-e_{\max }, \hat{\mu}+e_{\max }\right]$ with the probability of $p \geqslant 1-\alpha$ where $\hat{\mu}$ is the estimated mean value:

$$
e_{\max }=z_{\left(1-\frac{\alpha}{2}\right) ; n-1} \times \frac{\hat{\sigma}}{\sqrt{n}}
$$

where $z_{\left(1-\frac{\alpha}{2}\right) ; n-1}$ is the $1-\frac{\alpha}{2}$ quantile of the Student's $t$ distribution function with $n-1$ degrees of freedom, and $\hat{\sigma}$ is the estimated standard deviation. Moreover, the confidence interval for the standard deviation $\sigma$ can be determined by the estimated standard deviation $\hat{\sigma}$ as follows:

$$
\frac{\hat{\sigma} \sqrt{n-1}}{\chi_{\left(1-\frac{\alpha}{2}\right) ; n-1}} \leqslant \sigma \leqslant \frac{\hat{\sigma} \sqrt{n-1}}{\chi_{\left(\frac{\alpha}{2}\right) ; n-1}}
$$

where $\chi_{\left(1-\frac{\alpha}{2}\right) ; n-1}$ and $\chi_{\left(\frac{\alpha}{2}\right) ; n-1}$ are respectively the $1-\frac{\alpha}{2}$ and $\frac{\alpha}{2}$ quantiles of the chi-squared distribution with $n-1$ degrees of freedom. If the minimum number of samples $n_{\min }$ is unknown in advance, it can be obtained for a maximum acceptable error $e_{\max }$ and $\alpha_{\max }$ as follows:

$$
n_{\min }=\frac{z_{\left(1-\frac{\alpha_{\max }}{2}\right) ; n-1}^{2} \times \sigma^{2}}{e_{\max }^{2}}
$$

See the work by Krishnamoorthy ${ }^{34}$ for more details on statistical distributions and confidence intervals.

We determine the maximum number of samples $n_{\max }$ based on the overheads of the MC simulation on the time complexity of the analysis. Given the average execution times for construction $t_{c}$ and evaluation $t_{e}$ of the analysis core, a single analysis takes $t_{a}^{(1)}=t_{c}+t_{e}$. For $n$ samples, the analysis time equals $t_{a}^{(n)}=t_{c}+n \times t_{e}$ since the analysis core typically employs structures (BDDs, fault trees, Markov chains) that can be re-evaluated by assigning new values to their variables. Given the upper bound $\delta_{a}$ for the analysis time overhead, $n_{\max }$ can be calculated as follows:

$$
t_{a}^{\left(n_{\max }\right)} \leqslant\left(1+\delta_{a}\right) \times t_{a}^{(1)} \Rightarrow n_{\max }=1+\left\lfloor\frac{\delta_{a} \times t_{a}^{(1)}}{t_{e}}\right\rfloor
$$

To achieve a decent confidence level for the estimated statistical properties of uncertain objectives, the MC simulation uses 1000 samples, which results in an acceptable analysis time overhead according to the following section. However, the number of samples can be dynamically adapted if less confidence is required or time is restricted in the DSE. For an analysis with 1000 samples, a confidence level of $95 \%, z_{\left(1-\frac{\alpha}{2}\right) ; m} \approx 1.96$, can be achieved with a maximum estimation error of $e_{\max }<0.001$ according to equation (6). Note that more efficient sampling techniques such as Latin hypercube sampling ${ }^{35}$ can be used to reduce the minimum number of samples required to satisfy a desired confidence level.

Analysis overhead. Figure 8 shows the average construction time $t_{c}$ and evaluation time $t_{e}$ of the BDDs for different test problems on a logarithmic scale. As can be seen, $t_{c}$ is significantly larger than $t_{e}$, particularly when the degree of redundancy, the percentage of enabled redundant task-to-resource mappings, increases. This results from the $\mathrm{BDD}$ requiring exponential space in the number of variables in the worst case while a single evaluation can be done in linear time. While in existing approaches the BDD is typically constructed and evaluated once, we reuse the same BDD for multiple samples. For simple test cases, $t_{c}$ is about one to two orders of magnitude larger than $t_{e}$ such that for example 1000 samples increase the analysis time by a factor of 10 . 


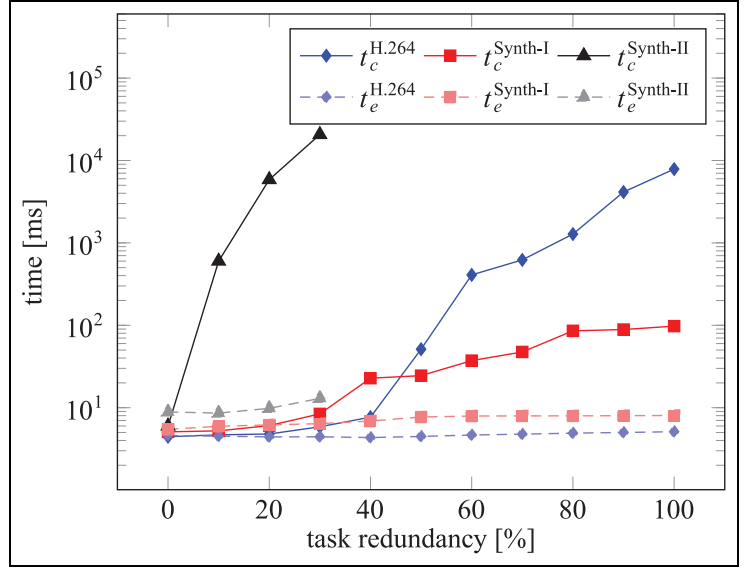

Figure 8. Construction $\left(t_{c}\right)$ and evaluation $\left(t_{e}\right)$ times of the $B D D$ for different test problems and redundancy degrees. Reported values are averaged from the analysis of 1000 random implementation candidates in each case. Note that the times are given in log-scale. Adapted from Khosravi et al. ${ }^{3}$

With an overall analysis time of around $1 \mathrm{~s}$, this is completely feasible for DSE. For more complex examples, $t_{c}$ is more than three orders of magnitude larger than $t_{e}$ such that for example 1000 samples do not even double the analysis time. From these results, it can be concluded that due to the construction overhead of the formal analysis core, the proposed analysis is still suitable for DSE, while for complex examples the overhead is minor compared to the insight gained.

Effects of ignoring correlation on reliability analysis. To verify the necessity of the proposed advanced correlation model in an uncertainty-aware design methodology, we study the effects of ignoring or simplifying the correlation model on the uncertainty distributions of the system's MTTF. To this end, we have measured different statistical properties, for example the mean value, of the system's MTTF calculated considering (i) the advanced correlation model, (ii) the simple correlation model, ${ }^{3}$ and (iii) no correlation. Comparing these properties, we can measure to what extent ignoring or underplaying the correlation model affects the uncertainty distributions, implying inaccuracy in their statistical properties. Such inaccuracies depend on characteristics of correlation, for example correlation degrees, among uncertain parameters in our model; in other words, ignoring a more extensive or stronger correlation may result in higher violation from the expected uncertainty distribution. To measure these inaccuracies, we introduce three correlation levels in Table 2 for the Synth-II test problem. These correlation levels differ in two aspects: (i) the number of groups wherein each parameter can be a member, and (ii) the degree to which a parameter can be correlated to a group.

Table 3 reports the average errors measured for the mean values and standard deviations, as well as bound intervals of the system's MTTF, notated respectively as
Table 2. Correlation levels. $|\Delta|$.

\begin{tabular}{lll}
\hline Correlation & & Degree \\
\hline barely & {$[0,2]$} & {$[0,0.33]$} \\
fairly & {$[1,4]$} & {$[0.33,0.66]$} \\
heavily & {$[2,5]$} & {$[0.66,1]$} \\
\hline
\end{tabular}

Table 3. Average errors, in percentages, caused by ignoring or simplifying correlation in the statistical parameters of MTTF objectives.

\begin{tabular}{lllll}
\hline Correlation & Model & $\overline{\mathrm{e}}_{\mu}$ & $\overline{\mathrm{e}}_{\sigma}$ & $\overline{\mathrm{e}}_{\mathrm{b}}$ \\
\hline barely & no & 0.07 & 5.86 & 10.15 \\
& simple & 0.07 & 5.95 & 10.35 \\
fairly & no & 0.24 & 41.63 & 43.53 \\
& simple & 0.25 & 42.21 & 43.94 \\
heavily & no & 0.44 & 79.73 & 79.29 \\
& simple & 0.43 & 79.07 & 78.73 \\
\hline
\end{tabular}

$\bar{e}_{\mu}, \bar{e}_{\sigma}$, and $\bar{e}_{b}$. As an example, for the simple correlation model, $\bar{e}_{\mu}$ can be calculated as follows:

$$
\bar{e}_{\mu}=\frac{1}{m} \sum_{i=1}^{m} \frac{\left|\mu_{i}^{\mathrm{adv}}-\mu_{i}^{\text {simple }}\right|}{\mu_{i}^{\text {adv }}}
$$

where $m=1000$ is the number of implementation candidates over which the average errors are calculated.

The results in Table 3 show that although neglecting correlation details does not impact the accuracy of the estimated mean values of MTTF, it may impose significant inaccuracies on the corresponding distributions. These inaccurate distributions can give the designer unrealistic views of the system's reliability which might lead to inappropriate design decisions.

Also, it can be seen in Table 3 that the analysis based on the simple correlation model might perform as badly as a correlation-oblivious analysis. Such underachievement of the simple model is due to the fact that a parameter with correlated uncertainty might behave noticeably differently when only one of its correlation groups is considered and the corresponding degree is set to a fixed value of 1.0. In other words, the corresponding samples obtained by simple and advanced correlation models are mostly from different parts of the parameter's uncertainty distribution. Note that different correlation sources are assumed to affect an uncertain parameter independently, that is, their quantile probabilities are sampled independently. Therefore, in the simple correlation model, the correlation sources whose effects on an uncertain parameter are ignored do not have any implicit impact on the samples taken from the parameter's uncertainty distribution.

\section{Conclusion}

Ever-shrinking device structures have made today's electronic systems susceptible to manufacturing 
tolerances as well as aging and radiation effects. The intensity of these effects cannot be precisely predicted since it depends on environmental conditions and usage profiles, and thus has to be considered an uncertain parameter in the design phase. Such uncertainties in different parts of the system can be correlated because destructive effects like temperature may affect several system components simultaneously. To deal with uncertainty in reliability analysis, our previous work ${ }^{3}$ combines existing analysis approaches with a MC simulation to consider diversely distributed uncertain parameters with multiple different correlations, and enables delivery of the uncertainty distribution of the system's MTTF accurately with an acceptable analysis time overhead. However, the simple uncertainty correlation model in Khosravi et al. ${ }^{3}$ fails to accurately analyze systems where the uncertainty of each component can be correlated with several uncertainty sources and to different degrees. To overcome such limitations, this paper presented a novel generalized correlation model that enables an accurate reliability analysis in systems with a lot of dependencies among uncertain parameters of their components.

\section{Declaration of Conflicting Interests}

The author(s) declared no potential conflicts of interest with respect to the research, authorship, and/or publication of this article.

\section{Funding}

The author(s) disclosed receipt of the following financial support for the research, authorship, and/or publication of this article: The work was supported in part by the German Research Foundation (DFG) as associated project CRAU (GL 819/1-2 \& TE 163/16-2) of the priority program Dependable Embedded Systems (SPP 1500).

\section{ORCID iD}

Faramarz Khosravi (iD https://orcid.org/0000-00019032-4751

\section{References}

1. Borkar S. Designing reliable systems from unreliable components: The challenges of transistor variability and degradation. IEEE Micro 2005; 25(6): 10-16.

2. Karl E, Sylvester D and Blaauw D. Analysis of systemlevel reliability factors and implications on real-time monitoring methods for oxide breakdown device failures. In: International symposium on quality electronic design (ISQED), 2008, pp. 391-395.

3. Khosravi F, Müller M, Glaß M, et al. Uncertainty-aware reliability analysis and optimization. In: Design, automation \& test in Europe (DATE), 2015, pp. 97-102.

4. Janicki M, Collet JH, Louri A, et al. Hot spots and coreto-core thermal coupling in future multi-core architectures. In: Semiconductor thermal measurement \& management symposium (SEMI-THERM), 2010, pp. 205-210.

5. Sun C, Shang L and Dick RP. Three-dimensional multiprocessor system-on-chip thermal optimization. In: International conference on hardware/software codesign \& system synthesis (CODES + ISSS), 2007, pp. 117-122.

6. Kaczer B, Mahato S, de Almeida Camargo VV, et al. Atomistic approach to variability of bias-temperature instability in circuit simulations. In: IEEE international reliability physics symposium (IRPS), 2011, pp. XT.3.1XT.3.5.

7. Amrouch H, van Santen VM, Ebi T, et al. Towards interdependencies of aging mechanisms. In: International conference on computer-aided design (ICCAD), 2014, pp. 478-485.

8. Lange A, Sohrmann C, Jancke R, et al. Multivariate modeling of variability supporting non-Gaussian and correlated parameters. IEEE Trans Comput-Aid Des Integ Circ Syst 2016; 35(2): 197-210.

9. Le Maître O and Kino O. Spectral methods for uncertainty quantification, with applications to fluid dynamics. Berlin: Springer, 2010.

10. Oberkampf W, Helton J, Joslyn C, et al. Challenge problems: Uncertainty in system response given uncertain parameters. Reliab Eng Syst Safe 2004; 85(1-3): 11-19.

11. Shang L, Peh LS, Kumar A, et al. Thermal modeling, characterization and management of on-chip networks. In: IEEE/ACM international symposium on microarchitecture (MICRO), 2004, pp. 67-78.

12. Ankireddi S. Robust prediction of critical temperatures in multi-core chips with limited sensory data. In: IEEE semiconductor thermal measurement \& management symposium (SEMI-THERM), 2011, pp. 216-221.

13. Zhang Y, Srivastava A and Zahran M. Chip level thermal profile estimation using on-chip temperature sensors. In: International conference on computer design (ICCD), 2008, pp. 432-437.

14. Chen $\mathrm{CH}$, Kim Y, Zhang Z, et al. A confidence-driven model for error-resilient computing. In: Design, automation \& test in Europe (DATE), 2011, pp. 1608-1613.

15. Coit DW, Jin $\mathrm{T}$ and Tekiner H. Review and comparison of system reliability optimization algorithms considering reliability estimation uncertainty. In: International conference on reliability, maintainability \& safety (ICRMS), 2009, pp. 49-53.

16. Herkersdorf A, Aliee H, Engel M, et al. Resilience articulation point (RAP): Cross-layer dependability modeling for nanometer system-on-chip resilience. Microelec Reliab 2014; 54(6-7): 1066-1074.

17. Souza FAL, Pereira P, De Paula H, et al. Motor drive systems reliability: Impact of the environment conditions on the electronic component failure rates. In: IEEE Industry Applications Society annual meeting, 2014, pp. 1-8.

18. Yin L, Smith $M$ and Trivedi K. Uncertainty analysis in reliability modeling. In: Reliability \& maintainability symposium (RAMS), 2001, pp. 229-234.

19. Esfahani N, Razavi K and Malek S. Dealing with uncertainty in early software architecture In: Foundations of software engineering (FSE), 2012, pp. 1-4.

20. Meedeniya I, Moser I, Aleti A, et al. Architecture-based reliability evaluation under uncertainty. In: Quality of software architectures (QoSA), 2011, pp. 85-94.

21. Sallak M, Schön W and Aguirre F. Transferable belief model for reliability analysis of systems with data 
uncertainties and failure dependencies. $J$ Risk Reliab 2010; 224(4): 266-278.

22. Ukhov I, Eles P and Peng Z. Temperature-centric reliability analysis and optimization of electronic systems under process variation. IEEE Trans VLSI 2014; 60(3): 667-674.

23. Teich J. Pareto-front exploration with uncertain objectives. In: Evolutionary multi-criterion optimization (EMO), 2001, pp. 314-328.

24. Limbourg P. Multi-objective optimization of problems with epistemic uncertainty. In: Evolutionary multicriterion optimization (EMO), 2005, pp. 413-427.

25. IEC 61709. Reference conditions for failure rates and stress models for conversion, 2011.

26. Karanki DR, Kushwaha HS, Verma AK, et al. Uncertainty analysis based on probability bounds (p-box) approach in probabilistic safety assessment. Risk Anal 2009; 29(5): 662-675.

27. Pedroni $\mathrm{N}$ and Zio E. Uncertainty analysis in fault tree models with dependent basic events. Risk Anal 2013; 33(6): 1146-1173.

28. Romberg W. Vereinfachte numerische integration. Kong Nor Vidensk Sels Forhand 1955; 28(7): 30-36.

29. Aliee H, Glaß M, Reimann F, et al. Automatic success tree-based reliability analysis for the consideration of transient and permanent faults. In: Design, automation \& test in Europe (DATE), 2013, pp. 1621-1626.

30. Zhan $\mathrm{Y}$ and Sapatnekar SS. High-efficiency green function-based thermal simulation algorithms. IEEE Trans Comput-Aid Des Integ Circ Syst 2007; 26(9): 16611675.

31. Blickle T, Teich $\mathbf{J}$ and Thiele L. System-level synthesis using evolutionary algorithms. Des Autom Embed Syst 1998; 3(1): 23-58.

32. Streichert T, Glaß M, Haubelt $\mathrm{C}$, et al. Design space exploration of reliable networked embedded systems. $J$ Syst Architec 2007; 53(10): 751-763.

33. Glaß M, Lukasiewycz M, Streichert T, et al. Reliabilityaware system synthesis. In: Design, automation \& test in Europe (DATE), 2007, pp. 409-414.

34. Krishnamoorthy K. Handbook of statistical distributions with applications. Boca Raton, FL: CRC Press, 2016.

35. McKay MD, Beckman RJ and Conover WJ. Comparison of three methods for selecting values of input variables in the analysis of output from a computer code. Technomet 1979; 21(2): 239-245.

\section{Appendix I}

\section{Notation}

$d$

$\Delta$

$\varepsilon$

$F^{-1}$

G

$g$

imp

$k$

$\mu$
$O_{A}$

$P$

$p$

$q_{0.95}$

$r$

$\rho$

$\mathcal{R}(t)$

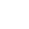

$\sigma$

$t$

$u$

$X^{l}$

$X^{u}$

\section{Appendix 2}

In this section we obtain PDFs for convolution of quantile probabilities and show its tendency towards a normal distribution. First, assume two independent random variables $q^{\prime}$ and $q^{\prime \prime}$ exist which follow the standard uniform distribution and represent quantile probabilities (for example $g_{j}$ and $u$ in equation (5)). Here we aim at calculating the PDF of the convoluted random variable $q$ which is defined as the weighted sum of $q^{\prime}$ and $q^{\prime \prime}$ :

$$
Q=d Q^{\prime}+(1-d) Q^{\prime \prime}
$$

Therefore, the PDF of $Q$ can be calculated using the following convolution integral:

$$
f_{Q}(x)=\int_{-\infty}^{+\infty} f_{d Q^{\prime}}(y) f_{(1-d) Q^{\prime \prime}}(x-y) \mathrm{d} y
$$

which results in

$$
f_{Q}(x)=\left\{\begin{array}{cc}
\frac{x}{d(1-d)}, & 0 \leqslant x<d \\
\frac{1}{1-d}, & d \leqslant x<1-d \\
\frac{1-x}{d(1-d)}, & 1-d \leqslant x \leqslant 1
\end{array}\right.
$$

when $d \leqslant \frac{1}{2}$, and

$$
f_{Q}(x)=\left\{\begin{array}{cc}
\frac{x}{d(1-d)}, & 0 \leqslant x<1-d \\
\frac{1}{d}, & 1-d \leqslant x<d \\
\frac{1-x}{d(1-d)}, & d \leqslant x \leqslant 1
\end{array}\right.
$$

when $d \geqslant \frac{1}{2}$. Examples for $f_{Q}(x)$ are shown in Figure 9(a) to (c). These PDF are averaged in Figure 9(d) to reflect the distribution of a correlated quantile probability $Q_{T}$, which corresponds to $\rho_{T}$ in equation (5). Applying weighted sum and averaging more often can result in even faster approximations of a normal distribution for $Q_{T}$. Such correlated quantiles cannot be applied to the two transformations in equations (3) and (4) since they include convolution of arbitrarily distributed uncertain parameters. However, our experiments 


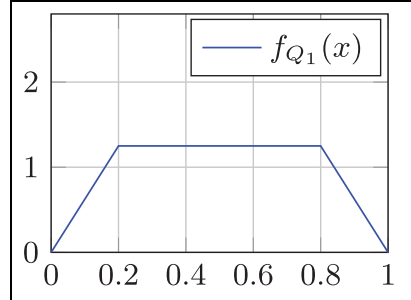

(a) $Q_{1}=0.2 Q^{\prime}+0.8 Q^{\prime \prime}$

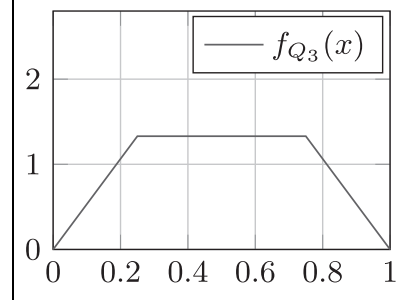

(c) $Q_{3}=0.75 Q^{\prime}+0.25 Q^{\prime \prime}$

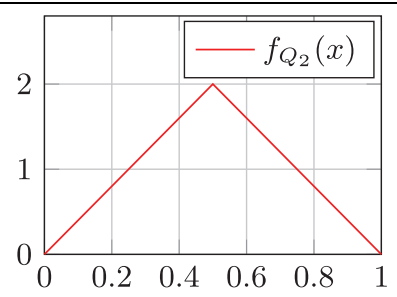

(b) $Q_{2}=0.5 Q^{\prime}+0.5 Q^{\prime \prime}$

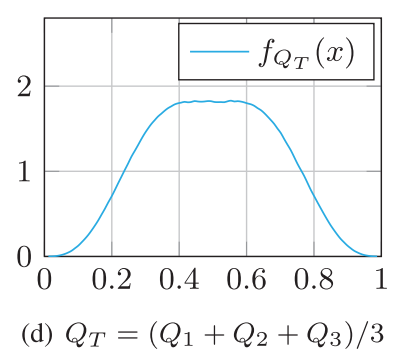

Figure 9. PDF of three random variables $q_{1}, q_{2}$ and $q_{3}$ representing weighted sums of two uniformly distributed random variables $q^{\prime}$ and $q^{\prime \prime}$, with different weights in (a) to (c), as described in equation (7). The average of these convoluted random variables indicates an approximation toward a Gaussian distribution in (d).

in Figure 4 indicate the tendency of all correlated distributions toward the original mean. In the following we show that in the boundary cases, where the correlation degrees approach 1 (no uniqueness) and the number of correlation groups goes to infinity, the correlated samples derived by each strategy in equations (3) to (5) would approach the mean value of the original distribution.

Theorem 1. Let the number of correlation groups of which uncertain parameter $p$ is a member go to infinity and the correlation degrees approach 1 . Then, the correlated samples yielded by each of the strategies in equations (3) to (5) of $p, E[p]$.

Proof. In the case of the first strategy in equation (3) the correlated sample can be approximated as follows:

$$
\begin{aligned}
& \rho_{\mathrm{I}}=\lim _{|\Delta| \rightarrow \infty} \frac{1}{|\Delta|} \sum_{i \mid G_{i} \in \Delta}\left(\lim _{d_{i} \rightarrow 1}\left(d_{i} F_{p}^{-1}\left(g_{i}\right)+\left(1-d_{i}\right) u\right)\right) \\
& =\lim _{|\Delta| \rightarrow \infty} \frac{1}{|\Delta|} \sum_{i \mid G_{i} \in \Delta} F_{p}^{-1}\left(g_{i}\right)
\end{aligned}
$$

Since $F_{p}^{-1}\left(g_{i}\right)$ points to a random point of the original distribution, averaging infinite random samples from a distribution will result in its expected value:

$$
\rho_{\mathrm{I}}=\lim _{|\Delta| \rightarrow \infty} \frac{1}{|\Delta|} \sum_{i \mid G_{i} \in \Delta} \rho_{i}=E[p]
$$

Given the second strategy in equation (4),

$$
\begin{aligned}
\rho_{\mathrm{II}} & =\lim _{|\Delta| \rightarrow \infty} \frac{1}{|\Delta|} \sum_{i \mid G_{i} \in \Delta} F_{p}^{-1}\left(\lim _{d_{i} \rightarrow 1}\left(d_{i} g_{i}+\left(1-d_{i}\right) u\right)\right) \\
& =\lim _{|\Delta| \rightarrow \infty} \frac{1}{|\Delta|} \sum_{i \mid G_{i} \in \Delta} F_{p}^{-1}\left(g_{i}\right)=E[p]
\end{aligned}
$$

Finally, using the last strategy in equation (5),

$$
\begin{aligned}
& \rho_{\mathrm{III}}=F_{p}^{-1}\left(\lim _{|\Delta| \rightarrow \infty} \frac{1}{|\Delta|} \sum_{i \mid G_{i} \in \Delta} \lim _{d_{i} \rightarrow 1}\left(d_{i} g_{i}+\left(1-d_{i}\right) u\right)\right) \\
& =F_{p}^{-1}\left(\lim _{|\Delta| \rightarrow \infty} \frac{1}{|\Delta|} \sum_{i \mid G_{i} \in \Delta} g_{i}\right)=F_{p}^{-1}(0.5)=E[p]
\end{aligned}
$$

Our experiments show that even with a small number of correlation groups and realistic degrees there exist inclinations among correlated samples toward $E[p]$. Intensifying the effects of groups and degrees increases the randomness of the correlated samples and make them converge toward a Gaussian distribution around a mean approximating $E[p]$. This convergence can be explained through the central limit theorem which states that the arithmetic mean of a sufficiently large number of independent random variables approximates a Gaussian distribution, regardless of the original distributions.

\section{List of Acronyms}

BDD binary decision diagram

$\mathrm{CCF}$ common-cause failure

CDF cumulative distribution function

DSE design space exploration

MC Monte Carlo

MTTF mean time to failure

NBTI negative bias temperature instability

NSGA-II non-dominated sorting genetic algorithm II

PDF probability density function 